\title{
The lung as a metabolic factory for gene therapy
}

\author{
John F. Engelhardt
}

Department of Anatomy and Cell Biology, Department of Internal Medicine, and Center for Gene Therapy of Cystic Fibrosis and Other Genetic Diseases, College of Medicine, The University of Iowa, Iowa City, Iowa, USA

J. Clin. Invest. 110:429-432 (2002). doi:10.1172/JCI200216443.

Over the past decade, the development of effective gene delivery vehicles to the lung has created numerous innovative strategies for treating inherited and acquired lung disorders. Currently, one of the most promising vectors for gene delivery to the lung is recombinant adenoassociated virus (rAAV). To date, gene therapy to the lung has focused on correcting genetic defects for diseases with intrinsic lung pathophysiology. One largely unexplored application of gene delivery to the lung is its use as a genetic metabolic factory for the production and systemic delivery of proteins into the blood for treatment of genetic diseases not directly associated with lung pathology. The study by Auricchio and coworkers described in this issue of the JCI focuses on this novel application (1). A conceptual extension of this work also includes the use of the lung as a catabolic factory for degradation of toxic, blood-borne molecules associated with genetic abnormalities in distant organs. Because the lung has one of the most expansive epithelial-blood capillary networks and is easily accessible for non-invasive gene delivery, it is uniquely suited for such applications.

\footnotetext{
Address correspondence to: John F. Engelhardt, Department of Anatomy and Cell Biology, The University of Iowa, College of Medicine, 51 Newton Road, Room 1-111 BSB, Iowa City, Iowa 52242, USA.

Phone: (319) 335-7744; Fax: (319) 335-7198;

E-mail: john-engelhardt@uiowa.edu.

Conflict of interest: The author's laboratory receives research support from Targeted Genetics Inc. but has no personal financial interests linked to this company.

Nonstandard abbreviations used: recombinant adeno-associated virus (rAAV); erythropoietin (Epo); alpha-1-antitrypsin (AAT); ornithine-delta-aminotransferase (OAT); methylmalonyl-CoA mutase (MCM).
}

\section{Genetic metabolic factories to treat genetic diseases}

The concept of metabolic factories for the ectopic production and delivery of genetically engineered protein products into the circulation is not new. Early reports of protein factories called organoids demonstrated therapeutic potential as self-contained genetic neo-organs for the delivery of secreted proteins into the blood (2). More recently, clinical trials in hemophilia B patients first attempted therapeutic delivery of factor IX to muscle (as an ectopic site for factor IX production) with encouraging results (3). Hemophilia $\mathrm{B}$ is an ideal disease for testing such approaches, since as little as $1 \%$ of the normal circulating factor IX protein level is likely to be therapeutic in hemophilia B patients (4). However, several concerns linger regarding the functional processing of factor IX in ectopic organs and are the impetus for new clinical trials directing expression of factor IX to the liver, its normal site of production.

rAAV-mediated delivery of transgenes to the lung, as employed by Auricchio et al., is advantageous in several respects. Ectopic expression of a blood-borne therapeutic protein can be limited by the immunogenicity of the vector or the transgene product. Immunogenicity to the vector may result in acute toxicity or may reduce transgene expression following repeated vector administration. In the context of $\mathrm{rAAV}$, the lung appears to be a far less immunogenic target than the muscle or liver. The lung is also uniquely suited for delivery of therapeutic proteins into the circulation because it offers a large number of target cells with good access to the blood for expressed proteins but not for the applied vector - the latter being a significant safety concern because of the possibility of inadvertent germ line delivery. Finally, the target cells in the lung appear competent to produce at least some therapeutic proteins with normal post-translational modifications and secretory properties, allowing them to be released into the bloodstream in a functional form.

The study described by Auricchio and colleagues demonstrates the therapeutic potential of using the lung as a genetic metabolic factory to produce and deliver proteins into the circulation. In this study they compared the efficiency of pseudotyped recombinant AAV1, 2, and 5 to deliver erythropoietin (Epo) and factor IX into the blood following lung gene transfer. Tests using alkaline phosphatase as a histochemical marker showed that rAAV2/5 was the most efficient in transduction of both the conducting airway epithelia, and alveolar regions of the lung and substantiated earlier work with this serotype in mouse lung (5). The efficiency of rAAV2/1 transduction was less than that for rAAV2 $/ 5$, but greater than that for rAAV2. This was the first report evaluating AAV Type 1 capsid for in vivo lung delivery and it appears to be similar in efficiency to its close relative AAV6 (6). The most interesting finding from this study, however, was the high efficiency with which Epo and factor IX were secreted from the airway epithelium into the circulation. Levels of factor IX in the blood were within the minimal range thought to be therapeutic for hemophilia B (1-2\% endogenous levels) and Epo levels also significantly increased hematocrit.

Given the fact that the airway epithelium is generally thought to secrete proteins predominantly into 


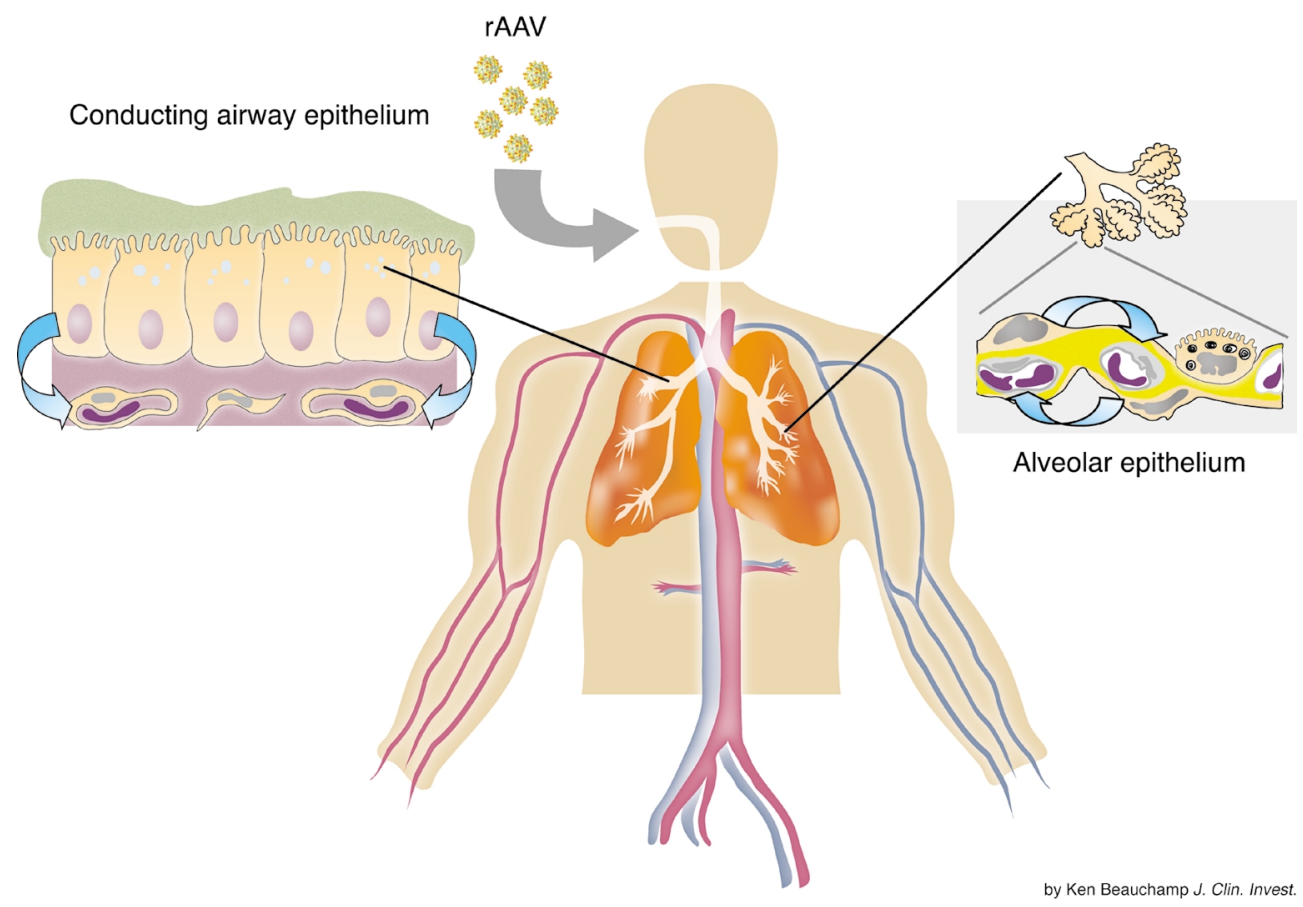

\section{Figure 1}

Using the lung as a metabolic factor for secretion of therapeutic proteins into the circulation. Vector administration to the lung might target the conducting airway epithelium, the alveolar epithelium, or both. Secretion of protein products from the basolateral membrane of epithelial cells must diffuse through the interstitium (composed of fibroblasts and connective tissue) and across vascular endothelial cells, to be delivered into the blood (as marked by arrows). The alveolar epithelia of the lung, which contain a prominent population of Type I pneumocytes, are in close proximity to the lung capillary network, making them ideal gene therapy targets for systemic delivery of proteins.

the lumen of the airways, these findings raise several fundamental questions regarding mechanisms that control polarity of protein secretion in the airway and their application to gene therapy. Interesting historical comparisons can be made to studies evaluating the polarity of alpha-1antitrypsin (AAT) secretion in polarized airway epithelial cells (7). These early studies demonstrated both apical and basolateral secretion of AAT from polarized airway epithelial cultures and suggested that gene transfer to the lung for AAT deficiency has the capacity to treat both airway and interstitial components of the disease. This present study by Auricchio et al., is the first in vivo proof of principal confirming these earlier in vitro findings. Although the level of apically secreted proteins was not evaluated in bronchial-alveolar lavages, given the functionally significant levels of transgene-derived protein in the blood, one can speculate that basolateral secretion of Epo and factor IX from the airway epithelia is quite efficient. Since conducting airway epithelia and alveolar epithelia were both targeted in the study by Auricchio, it is also currently unknown which of these two anatomical regions of the lung are responsible for the majority of transgene product delivery into the blood.

\section{Pulmonary immune tolerance to vector components}

Auricchio et al. also demonstrated in the present study that rAAV2/ 5 virus can be effectively re-administered to the lung. Although not evaluated, these findings suggest that the production of neutralizing antibodies to the AAV5 capsid may not present a major barrier to repeated dosing of the vector. It is currently unclear if the less immunogenic milieu of the lung is a generalized feature for repeat administration of all AAV serotype, since both findings of successful and unsuccessful repeat administration of rAAV2 in rabbit lung have been reported $(6,8)$. Although the functional significance of pre-existing antibodies to AAV is widely debated, there is a general consensus that repeat administration of rAAV to muscle and liver is hindered by neutralizing antibodies $(9-11)$. These findings suggest that the lung is less immunogenic from the standpoint of vector administration and also imply that transgene products may have reduced immunogenicity when ex-pressed in the lung. For example, the fact that factor IX levels persisted for 45 days in these studies suggests that neutralizing antibodies against canine factor IX may not have accumulated in mice. The ability of various organs to mount neutralizing antibodies to a neo-antigen such as factor IX is a significant issue for hemophilia B patients who carry null mutations in the gene for factor IX. Organ-specific dependencies in this regard have been found in mice where robust humoral responses against human factor IX are seen following intramuscular injection, but not portal vein injection to the liver, with lentiviral vectors (12). The relevance of these findings to the present study by Auricchio et al., which used canine factor IX, remains uncertain. Nevertheless, these findings cumulatively infer that the route of vector 
administration also affects the immunologic consequences to a transgene. In the case of the lung, these responses may be minimal.

\section{Lung anatomy, epithelial biology, and the polarity of secretion}

The lung is composed of two anatomically distinct regions including the conducting airways (including the trachea, bronchi, and bronchioles), and gas-exchanging airspaces (alveoli) (Figure 1). Each of these regions consist of distinct epithelial cell types with unique cellular physiologies. Since the most distal alveolar regions of the lung have the greatest access to the blood, it is likely the preferred target for protein delivery to the circulation. Two types of airway epithelia reside in this compartment, alveolar Type I and Type II cells. Type II alveolar cells represent the progenitor cell population in this compartment and hence would be the appropriate gene therapy target for persistent expression of integrated transgenes in this region of the lung. In contrast, Type I cells constitute the majority of surface area in the lung. This compartment is specially designed to have a large surface area-to-cell volume ratio that naturally promotes efficient diffusion of gas across the alveolar cells into the blood. Alveolar regions of the lung form approximately $100 \mathrm{~m}^{2}$ of surface area, of which Type I pneumocytes account for $-99 \%(13,14)$. This region is also highly vascular, with capillary endothelial cells accounting for $30 \%$ of total cells in the human lung (13). In contrast, the conducting airways constitute a minority of surface area in the human lung, amounting to approximately $0.25 \mathrm{~m}^{2}$ (15). Hence, one might anticipate that gene targeting to the alveolar epithelial cells (specifically the Type I pneumocyte) would hold the greatest potential for delivery of therapeutic proteins to the blood (Figure 1).

Most of the well studied synthesized proteins produced by polarized airway epithelia - mucus, antibacterial factors, surfactant proteins are apically secreted into the lumen of the airway. For a protein product to be delivered from lung epithelia to the blood, it must be secreted from the basolateral membrane. Results by
Auricchio et al. (1) and others (7), have demonstrated that factor IX, Epo, and AAT can all be secreted from the basolateral membrane of polarized airway epithelial cells. Because it is not clear that all other desirable therapeutic protein will share this property, molecular tags capable of redirecting secretory proteins from the apical to basolateral pathways could help improve the delivery of lung-produced proteins into the circulation.

\section{The lung as a catabolic factory?}

The present work establishing the lung as a metabolic factory for the production of therapeutic proteins in the circulation also raises the question as to whether gene expression in the lung could also be used as a catabolic factory to degrade circulating toxic metabolic products associated with genetic diseases, such as ornithine-delta-aminotransferase (OAT) and methylmalonyl-CoA mutase (MCM) deficiencies (Figure 2 ). In both of these examples, the accumulation of a toxic metabolic product, ornithine (in the case of OAT deficiency) or methylmalonate (in the case of MCM deficiency), results in disease pathology. Approaches for removing these toxic metabolic products from the circulation of affected individuals by gene therapy have been proposed and tested in experimental models $(16,17)$. The application of lung-directed gene therapy to catabolize toxic products from the circulation is an obvious extension of the work described by Auricchio and colleagues.

In summary, the lung has several inherent unique advantages as a potential therapeutic factory for the production, or degradation, of blood-borne molecules responsible for genetic disease. Most notably, gene delivery to the lung epithelium can be performed non-invasively with a large target cell population that has efficient access to the blood. Additionally, the lung possesses unique immunologic characteristics that may make it a preferred target for gene therapy from the standpoint of safety and repetitive vector-dosing. Despite the great promise of this approach to treat a subset of genetic diseases, several hurdles remain before its true potential will be realized. These include assessment and optimization of secretory properties for a given therapeutic protein and region of the airway, as well as the potential for abnormal protein processing in this organ which may alter the biologic properties of the protein being produced. These issues will likely be specific to a given therapeutic protein target.

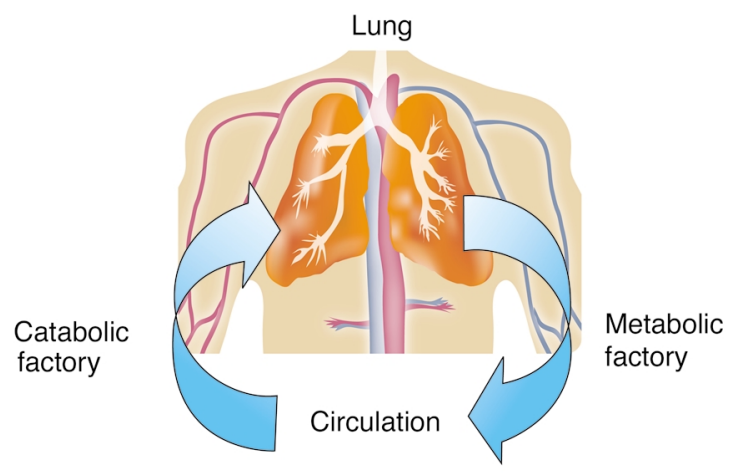

Figure 2

Using the lung as a genetically engineered metabolic or catabolic factory to treat genetic diseases. Gene transfer to the lung can theoretically be used to treat two classes of disorders associated with either the absence of a functional protein product or the accumulation of a toxic metabolite in the circulation. In the case of hemophilia B, the lung can be used as a metabolic factory to secrete factor IX into the circulation. In contrast, diseases for which a toxic metabolic intermediate accumulates in the circulation might also be treated by pulmonary expression of an enzyme that degrades the harmful compound. For example, ornithine-delta-aminotransferase expression in the lung could be used to degrade circulating ornithine, which is present in excess in patients with gyrate atrophy and results in blindness. 


\section{Acknowledgments}

Research in the author's laboratory for the areas discussed in this review is sponsored by NIH grant HL58340. The author also gratefully acknowledges Jude Gustafson for her editorial assistance.

1. Auricchio, A., et al. 2002. Noninvasive gene transfer to the lung for systemic delivery of therapeutic proteins. J. Clin. Invest. 110:499-504. doi:10.1172/JCI200215780.

2. Thompson, J.A., et al. 1989. Heparin-binding growth factor 1 induces the formation of organoid neovascular structures in vivo. Proc. Natl. Acad. Sci. USA. 86:7928-7932.

3. Kay, M.A., et al. 2000. Evidence for gene transfer and expression of factor IX in haemophilia B patients treated with an AAV vector. Nat. Genet. 24:257-261.

4. High, K.A. 2001. AAV-mediated gene transfer for hemophilia. Ann. NY Acad. Sci. 953:64-74.

5. Zabner, J., et al. 2000. Adeno-associated virus type 5 (AAV5) but not AAV2 binds to the apical surfaces of airway epithelia and facilitates gene transfer. J. Virol. 74:3852-3858.

6. Halbert, C.L., Rutledge, E.A., Allen, J.M., Russell, D.W., and Miller, A.D. 2000. Repeat transduction in the mouse lung by using adeno-associated virus vectors with different serotypes. J. Virol. 74:1524-1532.

7. Siegfried, W., et al. 1995. Polarity of secretion of alpha 1-antitrypsin by human respiratory epithelial cells after adenoviral transfer of a human alpha 1-antitrypsin cDNA. Am. J. Respir. Cell Mol. Biol. 12:379-384.

8. Beck, S.E., et al. 1999. Repeated delivery of adenoassociated virus vectors to the rabbit airway. J. Virol. 73:9446-9455.

9. Chirmule, N., et al. 2000. Humoral immunity to adeno-associated virus type 2 vectors following administration to murine and nonhuman primate muscle. J. Virol. 74:2420-2425.

10. Xiao, W., et al. 1999. Gene therapy vectors based on adeno-associated virus type 1.J. Virol. 73:3994-4003.

11. Moskalenko, M., et al. 2000. Epitope mapping of human anti-adeno-associated virus type 2 neutralizing antibodies: implications for gene therapy and virus structure. J. Virol. 74:1761-1766.

12. Park, F., Ohashi, K., and Kay, M.A. 2000. Therapeutic levels of human factor VIII and IX using
HIV-1-based lentiviral vectors in mouse liver. Blood. 96:1173-1176.

13. Crapo, J.D., Barry, B.E., Gehr, P., Bachofen, M., and Weibel, E.R. 1982. Cell number and cell characteristics of the normal human lung. Am. Rev. Respir. Dis. 126:332-337.

14. Harris, T.R., Waters, C.M., and Haselton, F.R 1994. Use of scaling theory to relate measurements of lung endothelial barrier permeability. J. Appl. Physiol. 77:2496-2505.

15. Mercer, R.R., Russell, M.L., Roggli, V.L., and Crapo, J.D. 1994. Cell number and distribution in human and rat airways. Am. J. Respir. Cell Mol. Biol. 10:613-624

16. Chang, C.C., Hsiao, K.J., Lee, Y.M., and Lin, C.M 1999. Towards metabolic sink therapy for mut methylmalonic acidaemia: correction of methylmalonyl-CoA mutase deficiency in T lymphocytes from a mut methylmalonic acidaemia child by retroviral-mediated gene transfer. J. Inherit. Metab. Dis. 22:773-787.

17. Sullivan, D.M., Jensen, T.G., Taichman, L.B., and Csaky, K.G. 1997. Ornithine-delta-aminotransferase expression and ornithine metabolism in cultured epidermal keratinocytes: toward metabolic sink therapy for gyrate atrophy. Gene Ther 4:1036-1044. 